OPEN ACCESS

Edited by:

Jason Roszik,

University of Texas MD Anderson

Cancer Center, United States

Reviewed by:

Franz Rödel,

Universitätsklinikum Frankfurt,

Germany

Katharina Stölzel,

Charité Universitätsmedizin Berlin,

Germany

${ }^{*}$ Correspondence:

Amanda Psyrri

psyrri237@yahoo.com

Specialty section:

This article was submitted to

Cancer Immunity and Immunotherapy,

a section of the journal

Frontiers in Oncology

Received: 14 July 2018 Accepted: 06 September 2018

Published: 26 September 2018

Citation:

Spathas N, Economopoulou P, Cheila M, Kotsantis I, Fanouriakis A,

Kassara D and Psyrri A (2018)

Inflammatory Arthritis Induced by Pembrolizumab in a Patient With Head and Neck Squamous Cell Carcinoma.

Front. Oncol. 8:409.

doi: 10.3389/fonc.2018.00409

\section{Inflammatory Arthritis Induced by Pembrolizumab in a Patient With Head and Neck Squamous Cell Carcinoma}

\author{
Nikolaos Spathas ${ }^{1}$, Panagiota Economopoulou ${ }^{1}$, Myrto Cheila ${ }^{1}$, loannis Kotsantis ${ }^{1}$, \\ Antonis Fanouriakis ${ }^{2}$, Dimitra Kassara ${ }^{2}$ and Amanda Psyrri ${ }^{1 *}$ \\ ${ }^{1}$ Section of Medical Oncology, Department of Internal Medicine, Faculty of Medicine, National and Kapodistrian University of \\ Athens, Attikon University Hospital, Athens, Greece, ${ }^{2} 4$ th Department of Internal Medicine, Rheumatology and Clinical \\ Immunology, Faculty of Medicine, National and Kapodistrian University of Athens, Attikon University Hospital, Athens, Greece
}

Introduction: T cell checkpoint inhibitors targeting Programmed cell Death protein-1 (PD-1) have emerged as novel immunotherapy agents showing remarkable efficacy in head and neck squamous cell carcinoma (HNSCC). Despite important clinical benefits, they are associated with side effects that occur as a consequence of general immunological stimulation due to loss of $T$ cell inhibition. Herein, we report the unusual case of inflammatory arthritis induced by anti-PD-1 agent pembrolizumab.

Case report: A 55-years old male was treated with pembrolizumab at a dose of $200 \mathrm{mg}$ every 3 weeks for a metastatic hypopharyngeal carcinoma. Following two cycles of immunotherapy, and while complete response of lung metastases was achieved, the patient presented with stiffness, swelling and pain of the right knee. Clinical examination and synovial fluid analysis revealed a seronegative inflammatory arthritis. Pembrolizumab therapy was interrupted and low-dose prednisone was administered with remarkable clinical improvement. Pembrolizumab was reintroduced, but after the fifth cycle, the patient developed inflammatory polyarthritis involving both knees and interphalangeal joints of both hands resulting in severe clinical deterioration. At that time, treatment with pembrolizumab was permanently discontinued. High-dose prednisone and methotrexate treatment led to remission of clinical symptoms.

Conclusion: Pembrolizumab-induced inflammatory arthritis is an unusual rheumatic immune-related adverse event that physicians are likely to encounter as $\mathrm{ICl}$ use expands. Multidisciplinary management and rheumatology consultation are necessary to provide immediate treatment and avoid permanent joint damage.

Keywords: pembrolizumab, inflammatory arthritis, head and neck cancer, immune checkpoint inhibitors, immunerelated adverse events 


\section{INTRODUCTION}

Immunotherapy has shown to provide durable responses for patients with advanced cancer (1). HNSCC serves as a paradigm of immunosuppressive disease, as it is characterized by dysregulated cytokine profile, impaired function of immune effector cells, and abnormalities in tumor-associated antigen (TAA) presentation (2). In November 2016, the Food and Drug Administration (FDA) approved nivolumab, an antiprogrammed cell death protein-1 (anti-PD-1) monoclonal antibody for the treatment of platinum-refractory recurrent and/or metastatic HNSCC based on a pivotal phase III clinical trial which demonstrated improved overall survival (OS) compared with chemotherapy (3). On the other hand, the antiPD-1 pembrolizumab has failed to improve OS in a phase III trial in the same setting (4).

Despite important clinical benefits, immunotherapeutic agents are associated with a wide spectrum of side effects termed immune-related adverse events (irAEs) that occur as a consequence of general immunological stimulation due to loss of $\mathrm{T}$ cell inhibition (5). Among irAEs, rheumatic and myoskeletal irAEs have to date not been widely characterized. Herein, we describe a case of inflammatory polyarthritis induced by pembrolizumab in a patient with metastatic HNSCC.

\section{CASE PRESENTATION}

A 55-years-old Caucasian male patient was diagnosed with a stage IVB head and neck squamous cell carcinoma (HNSCC) in May 2015. He was a heavy smoker and social drinker with no other significant medical history, and was initially treated with concurrent cisplatin-based chemoradiotherapy.
On routine follow-up visit in September 2016, Computed Tomography (CT) scans showed lung metastases. The patient was enrolled in a clinical trial and was randomized to pembrolizumab monotherapy every 3 weeks. Following the first two cycles of immunotherapy, the patient presented with stiffness, swelling and pain of the right knee. Physical examination showed inflammatory monoarthritis, with diffuse swelling and tenderness of the right knee. Laboratory tests were remarkable for an elevated erythrocyte sedimentation rate (ESR, $40 \mathrm{~mm} / \mathrm{h}$ ) and C-reactive protein (CRP, $50 \mathrm{mg} / \mathrm{L}$ ); rheumatoid factor (RF) and anti-cyclic citrullinated peptide (anti-CCP) antibodies were negative and serum uric acid was normal. Following rheumatologic consultation, knee joint aspiration was performed, and synovial fluid (SF) analysis revealed a yellow, cloudy appearance, decreased viscosity and a cell count of 7040 cells $/ \mathrm{mm}^{3}$ with $80 \%$ neutrophils, indicating an inflammatory arthritis. SF cultures were sterile and no crystals were found on microscopy. The patient was initially treated with prednisone $5 \mathrm{mg}$ twice a day with significant improvement over the following days. Inflammatory arthritis was attributed to pembrolizumab therapy and the third cycle was eventually postponed. Importantly, restaging imaging at that timepoint showed complete response of the disease.

Following reinstitution of pembrolizumab therapy, bilateral arthritis of the knees, accompanied by arthritis of interphalangeal joints of both hands (Figure 1A), developed after the fifth cycle, Ultrasound of the knees showed evidence of active synovitis (Figure 1B) and a diagnosis of inflammatory polyarthritis was established. The patient was retreated with prednisone $5 \mathrm{mg}$ twice a day and pembrolizumab therapy was interrupted. Due to the patient's clinical deterioration, and because protocol limitations did not allow increase of prednisone dose or administration of immunomodulatory drugs, pembrolizumab

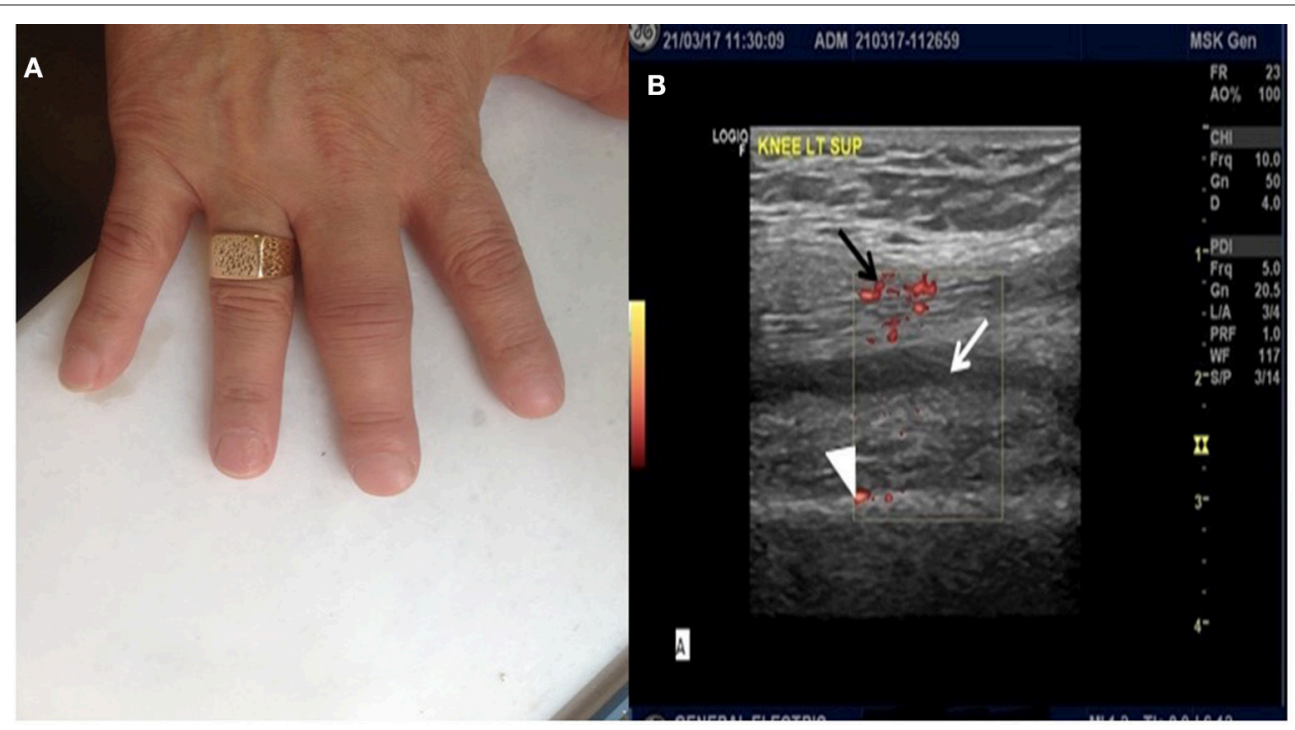

FIGURE 1 | (A) Arthritis of interphalangeal joints of the hands. (B) Ultrasound image of the left knee. Note the presence of synovial fluid (white arrow), marked synovial hypertrophy (white arrowhead) and presence of Power Doppler signal (black arrow), all indicative of active synovial inflammation. 
was permanently discontinued. Of note, the patient remained in complete remission. Methotrexate $7.5 \mathrm{mg}$ po as a single weekly dose was added to control synovial inflammation and, following also pembrolizumab discontinuation, the patient's symptoms gradually improved.

A written informed consent was obtained from the patient for the publication of this case report.

\section{DISCUSSION}

Immune-related adverse events (irAEs) induced by cancer immunotherapy have been described in a variety of clinical settings. Rheumatic and musculoskeletal events have been less often reported in clinical trials and most cases originate from case reports or case series (6-12). Our patient experienced severe symptoms, which severely compromised his quality of life. Interestingly, pembrolizumab retreatment triggered his symptoms.

In a recent report, Capelli and colleagues reviewed published literature on rheumatic and musculoskeletal irAEs. Arthritis was reported in 5/33 clinical trials, and vasculitis was reported in only 2. Case reports include the occurrence of inflammatory arthritis, vasculitis, myositis, and lupus nephritis (7). Of 13 patients with rheumatologic iRAEs following treatment with ipilimumab, or nivolumab in another series, 9 patients developed inflammatory polyarthritis (9). Consistent with our report, RF and anti-CCP antibodies were negative, albeit three cases were positive for ANA. Two more cases of delayed onset inflammatory polyarthritis after treatment with pembrolizumab for metastatic melanoma have been reported (12).

In a recent systematic review and meta-analysis of studies performed on cancer patients receiving anti-PD-1 and anti-PDL1 agents, only single cases of arthritis were reported in two studies at a rate of below $1 \%$ in each. However, across control groups, musculoskeletal complaints were very common, ranging from 9 to $18 \%$ for arthralgia, 2 to $16 \%$ for back pain, 4 to $6 \%$ for musculoskeletal pain, and 4 to $16 \%$ for myalgia (13). In another retrospective study that included 496 patients with metastatic

\section{REFERENCES}

1. Finn OJ. Immuno-oncology: understanding the function and dysfunction of the immune system in cancer. Ann Oncol. (2012) 23 (Suppl. 8):viii6-9. doi: 10.1093/annonc/mds256

2. Ferris RL, Whiteside TL, Ferrone S. Immune escape associated with functional defects in antigen-processing machinery in head and neck cancer. Clin Cancer Res. (2006) 12:3890-5. doi: 10.1158/1078-0432.CCR-05-2750

3. Ferris RL, Blumenschein G Jr, Fayette J, Guigay J, Colevas D, Licitra L, et al. Nivolumab for recurrent squamous-cell carcinoma of the head and neck. N Engl J Med. (2016) 375:1856-67. doi: 10.1056/NEJMoa16 02252

4. Cohen EE, Harrington KJ, Le Tourneau C, Dinis J, Licitra L, Ahn M, et al. Pembrolizumab (pembro) vs. standard of care (SOC) for recurrent or metastatic head and neck squamous cell carcinoma (R/M HNSCC): Phase 3 KEYNOTE-040 trial. Ann Oncol. (2017) 28 (suppl.5): v605-49. doi: $10.1093 /$ annonc/mdx440

5. Uetrecht J. Immune-mediated adverse drug reactions. Chem Res Toxicol. (2009) 22:24-34. doi: 10.1021/tx800389u melanoma who were treated with anti-PD-1 agents, arthritis was rarely reported $(14,15)$.

The etiology of pembrolizumab-induced inflammatory arthritis is not yet clarified. It has been suggested that antiPD1 antibodies enhance autoimmunity by activating $\mathrm{T}$ cell function and may allow previously dormant arthritogenic clones to expand or newly presented autoantigens to develop into arthritogenic clones (12). Indeed, there might be a correlation between enhanced autoimmunity and response to immunotherapy treatment. Vitiligo has been correlated with increased immune response in patients receiving pembrolizumab (16). This is consistent with our case, which developed severe arthritis, but also complete disease remission.

Our case is unique in that the patient experienced severe symptoms that remarkably affected his quality of life; of note, his cancer did not cause him any symptoms. Furthermore, complete remission was achieved and remained after pembrolizumab discontinuation. We believe that severity of inflammatory arthritis correlated with increased immune response.

At present, our knowledge about the incidence and the diagnosis of rheumatic irAEs is limited. Furthermore, no consensus exists regarding the treatment of arthritis in this setting. High dose glucocorticoids might alter the efficacy of immunotherapeutic agents and immunomodulatory drugs might trigger tumor progression. Frequently, discontinuation of immunotherapy remains the only option. Although optimal duration of immunotherapy treatment has not been established in cancer, it is uncertain whether the efficacy of these drugs persists after their discontinuation. Collaboration between rheumatologists and oncologists is pivotal in order to understand the spectrum of rheumatic irAEs, optimize their treatment and define the appropriate time point of treatment discontinuation, if necessary.

\section{AUTHOR CONTRIBUTIONS}

All authors listed have made a substantial, direct and intellectual contribution to the work, and approved it for publication.

6. Belkhir R, Burel SL, Dunogeant L, Marabelle A, Hollebecque A, Besse B, et al. Rheumatoid arthritis and polymyalgia rheumatica occurring after immune checkpoint inhibitor treatment. Ann Rheum Dis. (2017) 76:1747-50. doi: 10.1136/annrheumdis-2017-2 11216

7. Cappelli LC, Gutierrez AK, Bingham CO III, Shah AA. Rheumatic and musculoskeletal immune-related adverse events due to immune checkpoint inhibitors: a systematic review of the literature. Arthritis Care Res. (2016) 69:1751-63. doi: 10.1002/acr.23177

8. Cappelli LC, Shah AA, Bingham CO III. Immune-related adverse effects of cancer immunotherapy- implications for rheumatology. Rheum Dis Clin North Am. (2017) 43:65-78. doi: 10.1016/j.rdc.2016. 09.007

9. Cappelli LC, Gutierrez AK, Baer AN, Albayda J, Manno RL, Haque $U$, et al. Inflammatory arthritis and sicca syndrome induced by nivolumab and ipilimumab. Ann Rheum Dis. (2017) 76:43-50. doi: 10.1136/annrheumdis-2016-209595

10. Gauci ML, Baroudjian B, Laly P, Madelaine I, Da Meda L, Vercellino L, et al. Remitting seronegative symmetrical synovitis with pitting edema (RS3PE) 
syndrome induced by nivolumab. Semin Arthritis Rheum. (2017) 47:281-7. doi: 10.1016/j.semarthrit.2017.03.003

11. Calabrese C, Kirchner E, Kontzias K, Velcheti V, Calabrese LH. Rheumatic immune-related adverse events of checkpoint therapy for cancer: case series of a new nosological entity. RMD Open (2017) 3:e000412. doi: 10.1136/rmdopen-2016-000412

12. Chan MM, Kefford RF, Carlino M, Clements A, Manolios N. Arthritis and tenosynovitis associated with the anti-PD1 antibody pembrolizumab in metastatic melanoma. J Immunother. (2015) 38:37-9. doi: 10.1097/CJI.0000000000000060

13. Baxi S, Yang A, Gennarelli RL, Khan N, Wang Z, Boyce L, et al. Immunerelated adverse events for anti-PD-1 and anti-PD-L1 drugs: systematic review and meta-analysis. BMJ (2018) 360:k793. doi: 10.1136/bmj.k793

14. Sosa A, Lopez Cadena E, Simon Olive C, Karachaliou N, Rosell R. Clinical assessment of immune-related adverse events. Ther Adv Med Oncol. (2018) 10:1758835918764628. doi: $10.1177 / 1758835918764628$

15. Zimmer L, Goldinger SM, Hofmann L, Loquai C, Ugurel S, Thomas I, et al. Neurological, respiratory, musculoskeletal, cardiac and ocular side-effects of anti-PD-1 therapy. Eur J Cancer (2016) 60:210-25. doi: 10.1016/j.ejca.2016.02.024

16. Hua C, Boussemart L, Mateus C, Routier E, Boutros C, Cazenave H, et al. Association of vitiligo with tumor response in patients with metastatic melanoma treated with pembrolizumab. JAMA Dermatol. (2016) 152:45-51. doi: $10.1001 /$ jamadermatol.2015.2707

Conflict of Interest Statement: The authors declare that the research was conducted in the absence of any commercial or financial relationships that could be construed as a potential conflict of interest.

Copyright $\odot 2018$ Spathas, Economopoulou, Cheila, Kotsantis, Fanouriakis, Kassara and Psyrri. This is an open-access article distributed under the terms of the Creative Commons Attribution License (CC BY). The use, distribution or reproduction in other forums is permitted, provided the original author(s) and the copyright owner(s) are credited and that the original publication in this journal is cited, in accordance with accepted academic practice. No use, distribution or reproduction is permitted which does not comply with these terms. 